This item was submitted to Loughborough's Research Repository by the author.

Items in Figshare are protected by copyright, with all rights reserved, unless otherwise indicated.

\title{
Twitter and global political crises
}

\section{PLEASE CITE THE PUBLISHED VERSION}

https://doi.org/10.1163/18739865-01002006

\section{PUBLISHER}

(c) Brill

\section{VERSION}

AM (Accepted Manuscript)

\section{PUBLISHER STATEMENT}

This paper was accepted for publication in the journal Middle East Journal of Culture and Communication and the definitive published version is available at https://doi.org/10.1163/18739865-01002006

\section{LICENCE}

CC BY-NC-ND 4.0

\section{REPOSITORY RECORD}

O'Loughlin, Ben, Cristian Vaccari, Billur A. Ozgul, and James Dennis. 2017. "Twitter and Global Political Crises". figshare. https://hdl.handle.net/2134/28256. 


\section{Twitter and Global Political Crises.}

\section{Cycles of Insecurity in \#PrayforParis and \#PrayforSyria}

\section{Ben O'Loughlin}

Royal Holloway, University of London, uk

Corresponding author: Ben.OLoughlin@rhul.ac.uk

\section{Cristian Vaccari}

Royal Holloway, University of London, uk

\section{Billur Aslan Ozgul}

Royal Holloway, University of London, uk

\section{James Dennis}

University of Portsmouth, uk

\section{Introduction}

This article examines public reactions on social media to the 13 November 2015 attacks by Islamic State on Paris that were driven by the Twitter hashtag \#PrayforParis and the response from those adopting a \#PrayforSyria hashtag. The latter appeared to contest the exclusive or primary attention of Twitter users to conflict in Paris over conflict in Syria and elsewhere. We gathered nearly three million tweets containing the hashtags \#prayforparis and \#prayforsyria in the days after the attack. As a research team observing news media coverage of the attacks and apparently backlashes against Muslims and/or migrants in Europe, we expected to find debate around these hashtags would contain users conflating three issues around the attacks: (i) migration: were the attackers homegrown or carrying overseas passports? (ii) violence: why was Paris attacked and why is France bombing Islamic State? (iii) media: what role should mainstream media and social media play during such events? In the event's aftermath, debates raged about whether news media and social media platforms like Facebook offer disproportionate attention to casualties in Paris (See Figure 1 below) 
when catastrophes were unfolding simultaneously in Lebanon, Japan and elsewhere. Such debates condition immediate public and policy responses - the backlash - but also shape how public spheres function in the long term.

Mark Zuckerberg $\odot$ Many people have rightfully asked why we turned on Safety Check for Paris but not for bombings in Beirut and other places.

Until yesterday, our policy was only to activate Safety Check for natural disasters. We just changed this and now plan to activate Safety Check for more human disasters going forward as well.

Here's more detail on Safety Check and our policy for deploying it from the Facebook Safety page:

https://www.facebook.com/fbsafety/posts/930229667014872

Thank you to everyone who has reached out with questions and concerns about this. You are right that there are many other important conflicts in the world.

We care about all people equally, and we will work hard to help people suffering in as many of these situations as we can.

Figure 1. Facebook CEO Mark Zuckerberg explains Facebook’s Safety Check policy

The aim of this study is to identify and explain the main differences in the contents, sources, and diffusion networks of the tweets about the Paris and Syria attacks. We build on previous research exploring how social media affordances encourage certain communication behaviours. We make two empirical contributions. First, we operationalise complex and fuzzy concepts from political and social theory through systematic descriptive, bivariate and multivariate analysis of public communication. We first test the hypothesis that the \#PrayforSyria Tweets were more argumentative. We know from political theory that presenting an argument in public necessarily makes one vulnerable or exposed - to confrontation, misrepresentation, or simply being ignored (Cavarero 2000). We know from social theory that tweeting in more directly confrontational ways carries a higher affective demand than simply sharing other people's information (Miller, 2012). It involves greater “identity work”: one risks one’s “face” in posting a politically-charged statement or directly posting a comment to an original commenter through Twitter's reply function. We conceptualise argumentative behaviour through the concepts agonism and antagonism (Mouffe, 2000). Antagonism refers to statements that seek to exclude others from the shared space of politics: to either kill people or drive them out of the debate. Agonism refers to 
statements that dispute a policy or perspective but do not seek the exclusion or destruction of those they disagree with. Agonism and Antagonism are processes, not end-points; they are forms of action that constitute the endless play of claim and counter-claim that defines "the political”. We expect to find more agonism and antagonism in \#PrayforSyria tweets than \#PrayforParis because, drawing on the "pray for Syria" trope that had been present in Syrian Facebook discussions since 2013 (Crilley, 2016), users remixed it as a hashtag with the apparent motivation to contest the distribution of global attention and hierarchy of pity (Boltanski, 1999). However, given the conflation of discussion of media, migration and terrorism in European news media itself (Berry et al., 2015), we test the second hypothesis that users conflate discussion of media, migration and terrorism.

The second empirical contribution, then, is to explain the drivers of agonism and antagonism. We do this by testing the degree to which agonism and antagonism are correlated with retweet volumes, topic of debate, location, and type of user. We take retweets as key indicators: retweets refer to the act of sharing another user's tweet to their own followers by clicking the RT symbol that accompanies that tweet. Retweets indicate the popularity of a tweet. We analyse them as dependent variables because this explains what properties of tweets are more likely to be popular and spread. Among replies to less-retweeted posts we expect to find less antagonism. However, regards hypothesis two, we also explore whether the conflation of media, migration and terrorism drives the presence of agonism and antagonism.

This article makes two theoretical contributions. First, this research helps us understand how ethics work in a global digital ecology. The shift from broadcast to Web 2.0 offers interaction and thus those suffering can be present with us, not presented to us (Miller, 2012). Floridi argues: 'we are witnessing a substantial erosion of the right to ignore' (2014: 42). He continues: 'The more any bit of information is just an easy click away, the less we shall be forgiven for not checking it. ICTs are making humanity increasingly responsible, morally speaking, for the way the world is, will be, and should be' (2014: 42-3). Nevertheless, our digital communities still have boundaries. Some remain invisible to us. Consequently, some feel the need to make connectivity, to bring those suffering outside our networks to presence within them. The \#PrayforSyria promotion was one such effort: to bring another set of suffering humans to visible presence and recognition. Our study is set against a context in which hyperconnectivity could cultivate a new mass responsibility. How are audiences to 
compare and evaluate several tragedies? Was \#PrayforSyria making the point that it is possible to care about more than one tragedy at once, or to bring balance between Western and non-Western tragedies, or some other motive?

Second, this research illuminates some new patterns in social responses to trigger events such as terrorist attacks. Our conception of 'cycles of insecurity' builds on a trajectory of sociological research on the role of mainstream media in amplifying moral panics.

Particularly in the wake of disruptive social and political events, news reporting often demonises certain social groups as 'folk devils' in ways that validated existing prejudices against such groups in ways that feed into communicative and even physical violence against them, triggering further rounds of counter-attacks and reprisals (Cohen, 1972; Hall et al., 1978; Poynting et al., 2004). For several decades, this theory was relatively settled because the public sphere was anchored by television, radio and press. However, criminologists have now begun to explore how social media enable different types of dynamics that amplify hate speech and possibly violence. Williams and Burnap (2016) studied public responses on social media to the London 2013 terrorist attack that saw the murder of soldier Lee Rigby. They applied Cohen's moral panics theory to identify degrees of hate speech in the initial impact stage before police and mainstream media had confirmed what was happening. Analysing 15 days of Tweets following the event, they found it was only in that initial few hours, before the “terrestrial' paced police response' could impose official meaning, that hate speech appeared with any frequency (ibid., 234); the police response soon stifled any cycle of insecurity and racist backlash. Importantly, Williams and Burnap found evidence of 'countercyberhate speech’ (ibid., 233). Social media allow individuals to openly express this desire for positive intercultural relations and minimisation of antagonism. For example, after the Brussels attacks in March 2016 the \#StopIslam hashtag emerged as a Twitter trending topic around the world but its surge in frequency was due to people contesting it and condemning racism (Dewey, 2016).

This study explores new mechanisms through which publics and counter-publics form and seek to make issues visible and themselves recognisable as publics making claims (Barnett, 2003; Warner, 2002). These new patterns are not only of interest for theorising media and insecurity. They can also be expected to feed into all actors' assumptions about how communication patterns will unfold after terrorist attacks or other catastrophes. In this way, 
the 'cycles of insecurity' acquire altered dynamics that will affect perceptions and behaviour. Hence, it is vital we understand how antagonism is generated.

\section{Mass responses on social media: Shifting from emotion to antagonism}

Studies evaluating emotion in politics in social media content have tended to use sentiment binaries rather than scales. Either an emotion is present/absent or emotion is positive/negative. Human-coded studies of emotion on Twitter, particularly since the Arab Uprisings, have focused on one particular emotion per study, for instance 'enthusiasm' or 'feeling of drive'. These offer little conceptual validity. Moreover, there are no systematic studies of political contestation in user interactions, such as individuals' use of \#PrayforSyria to make claims about Syria's relation to the Paris attacks. This warrants a turn to theories of political contestation. In this section we define an antagonistic tweet in contrast to an agonistic tweet.

Antagonism is associated with emotion, and emotion affects behavior online. Several studies have found that valence (strong positive/negative sentiment) and arousal (high/low excitement) associates with increased sharing of content on social media by users (Berger, 2011; Berger and Milkman, 2012; Larson et al, 2011). Researching the US movement Black Lives Matter and \#Blacklivesmatter hashtag, Freelon et al (2016) coded tweets as supportive, opposed or non-aligned to the movement. They used interviews with movement activists to explain whether certain Twitter surges were motivated by emotions such as anger. They did not code emotion or antagonism in tweets in any transparent or granular manner.

Two major studies of social media emotion again miss contestation. Meraz and Papacharissi (2013) analysed 959,893 Latin character Tweets from the month beginning 24 January 2011 when protests began in Cairo calling for President Mubarak to resign. The validity of coding for emotion was undermined because they found fact and emotion difficult to disentangle: 'most tweets were not just news or just opinion, but typically a blend of emotionally charged opinions on news' (ibid: 156). As certain charged pieces of news became retweeted, so they took on the function of refrains analogous to those found in music, marking 'an intensity that provides the pulse for a growing movement' (ibid: 156). They then used qualitative analysis - 'notes taken regarding language use and tone' - to document what they labeled 'a feeling of ongoing drive' as users expressed anticipation and excitement about a revolution to come (ibid: 148, 160). Gerbaudo (2016) builds on Meraz and Papacharissi's study by seeking to 
quantify these emotional dynamics. Taking the protest Facebook pages of KKS (We are all Khaled Said) in the 2011 Egyptian revolution and the DRY (Real Democracy Now) page from Spanish protests of the same year, Gerbaudo identifies what he calls 'moments of digital enthusiasm'. These are defined as 'necessarily transient phases of intense, positive emotional mood emerging in political online conversations in proximity to major protest events' (2016: 255). Quantitative analysis illustrated increased frequency of likes and comments. These data stood as proxies for emotion and attention around key events. Qualitative analysis of comments focused on tone and the use of exclamation marks, capitalized words and emoticons.

We must turn to the emergent hate speech literature for conceptualisation of what antagonism looks like on Twitter. In their review of existing studies, Burnap and Williams (2016: 5) find the presence of hate and antagonism have been measured in terms of use of profane or pejorative words, use of first and second person pronouns, use of accusational terms, and use of 'othering' language that presents a 'we-they' dichotomy on religious or other grounds and is derogatory about the 'they'. Through computational linguistics and the use of multiple human coders they are able to analyse these grammars and vocabularies with high reliability. Despite these tools, however, they ultimately only evaluate whether such speech is present or absent. Equally, in their study of responses to the 2013 Lee Rigby terrorist attack they measure a Tweet's sentiment as either positive, negative or neutral (Williams and Burnap, 2016). Measuring antagonism and the emotions surrounding antagonism on a spectrum or scale - quite antagonistic, very antagonistic - is too complex even for computational methods and large human teams.

For this reason, as a small-scale human-coding project, we look for the presence of antagonism and agonism and see how these associate with retweeting and other observable user behavior. We draw upon Mouffe's conception of democratic talk proceeding through agonism, in which one considers one's political opponent at least to be a legitimate adversary rather than, in antagonistic talk, an enemy to be eliminated (Mouffe, 1993; 2000). For Mouffe, politics involves articulatory practices linking or not linking certain issues and identities, for instance those seeking to link migrants, terrorism and the internet as a way to draw boundaries between a threatened "us" and dangerous "them”. There is no possibility of resolution because the political principles under dispute, such as freedom of movement, physical security and equal rights, cannot all be fully realized. This 'undecidability' keeps 
politics open (Mouffe, 1992a: 13). The political space, for Mouffe, is a community held together by 'public concern', not by an idea, network shape, or identity (Mouffe, 1992b: 233). A Twitter conversation cohered by a hashtag enables that focus of public concern. While we expect most users to find a limit to the space of those sharing their opinion, in finding that limit and being exposed to other views, users realise the contestability of the issue (cf. Laclau and Mouffe, 1988, 93-148).

We extend Mouffe's conception to include the referent. We do so in a way consistent with Schmittian aspect of Mouffe's thought, namely that politics involves a firm line between friend and enemy (Mouffe, 1993; Schmitt, 1976). In an agonistic tweet, the user considers those being discussed - migrants, refugees, ethnic or religious minorities - a legitimate adversary whose place within democratic society can be acknowledged. In an antagonistic tweet, the user can express antagonism not just in response to another user's tweet, but by considering referents being discussed - again migrants, refugees, ethnic or religious minorities - as outside the legitimate polity: to be arrested, expelled and/or killed.

\section{Methodology}

This research is based on qualitative and quantitative analysis of Tweets for the five days following the Paris attacks of 13 November 2015. The research question and hypotheses driving this study are:

RQ1: What are the main differences in the contents, sources, and diffusion networks of the tweets about the Paris and Syria attacks?

H1: Agonism and antagonism are driven by a conflation of media, migration and terrorism. H2: Antagonism is associated with retweet volumes: Tweets that are antagonistic are more likely to be retweeted.

Our workflow model to answer this question and test these hypotheses is: 
i. We gathered all Tweets containing the hashtags \#PrayforParis $(N=2,088,075)$ and \#PrayforSyria $(N=829,053)$ via Twitter's Search API ${ }^{1}$ in the time period 1318 November $2015 .^{2}$

ii. We cleaned the data by removing retweets, leaving a total of 269,075 unique tweets containing \#PrayforParis and 56,618 unique tweets containing \#PrayforSyria. ${ }^{3}$

iii. Four coders agreed and discussed the coding frame in serial meetings. To guarantee reliability in our coding, we extracted random samples of tweets, ensuring that they contained an equal proportion of \#PrayforParis and \#PrayforSyria tweets, and each coder coded them independently. We ran four iterations of this procedure ( $N=100$ tweets in the first two rounds, $N=50$ in the last two rounds), excluding at each round the variables for which we had achieved satisfactory reliability scores (which we report in the appendix). ${ }^{4}$ We excluded two variables from the original coding framework - the main purpose of the tweet and whether the tweet contained references to solidarity - because we did not achieve satisfactory reliability scores even after four iterations.

iv. We subsequently generated a random sample $(N=4,500)$ that included an equal number of tweets from each corpora and coded it according to our finalized coding framework. We did not code a total 152 tweets that we identified as spam.

\footnotetext{
${ }^{1}$ API stands for "Application Programming Interface" and is a set of instructions and protocols that enables users to access a web-based software application. Twitter's API (https://dev.twitter.com/) allows researchers to retrieve public Twitter messages as well as relevant "metadata" such as the identifier of the user who posted them, date, location, language, and so forth. The Search API allows searching tweets posted during the previous week. We limited our search to tweets posted by users using English as the language of their Twitter interface so that most of the tweets we collected could be expected to be in English. To minimise loss of data due to technical errors, we ran multiple searches in parallel using the same keywords and subsequently combined the results from all searches, deleting duplicate messages we identified based on the unique identification number that Twitter assigns to each tweet when published.

${ }^{2}$ The first tweet in our collection was posted at 23:27 Paris time on 13 November 2015, about two hours after the reported occurrence of the first bombing. The last tweet was posted a few seconds before midnight on 18 November 2015.

${ }^{3}$ As readers may note, excluding retweets left us with a relatively smaller number of tweets containing \#PrayforSyria compared with \#PrayforParis than in the original collection. This is because, as we shall see below, the tweets about Syria were substantially more likely to be retweeted than those about Paris.

${ }^{4}$ To assess our intercoder reliability we used two indices: Krippendorff's alpha and average percent pairwise agreement. Given the exploratory nature of our research, we accepted as reliable the variables that achieved Krippendorrff's alpha scores equal to or higher than .667 (as suggested by Krippendorff, 2004). For some dichotomous variables with heavily skewed distributions, we followed Bennett et al. (2011) and relied on percent agreement because in these instances measures such as Krippendorff's alpha tend to underestimate intercoder reliability (see Neuendorf, 2002). In those instances, we treated variables where we had achieved percent agreement of $90 \%$ or above as reliably coded.
} 
v. We ran univariate, bivariate and multivariate quantitative analyses on the dataset combining the variables we coded and some of the meta-data obtained from Twitter.

vi. We tested inferences deriving from our quantitative analysis against small samples of relevant tweets.

Our coding framework (see appendix) covered (A) type of actor, (B) location of actor, (C) type of tweet, (D) type of content shared, (E) attacks mentioned - Paris, Syria, or Elsewhere -, (F) topics mentioned - migration, foreign policy, terrorists, religion, mainstream media, social media, justice and fairness, and universalism -, and (G) agonism and antagonism. It was designed to identify the presence of agonism and antagonism and their associations with who is tweeting, how, and about what. We also gathered data on the numbers of friends and followers of each user in our sample and the retweet volume of each tweet.

Given the exploratory nature of our research and the granularity of the concepts we wanted to measure, we relied on human coding not computational methods. Dictionary-based methods would have been very difficult to employ given the complexity of these topics and the fact that we aimed at comparing two different conversations that, as we will show, centred upon different topics. Although using machine learning techniques was a possibility for textual analysis, many tweets contained images and/or links to external websites, which we included in our content analysis and which would have been very difficult to train a computational tool to automatically analyse (for an overview of these methodologies see Ampofo et al., 2015).

\section{Analysis 1: Overall Differences Between Tweets Mentioning Paris and Syria}

We begin our analysis by comparing tweets mentioning the Paris and the Syria attacks in terms of their sources, contents, and network diffusion. A total of 2,639 tweets (61\% of the 4,348 we coded) mentioned Paris while 2,164 (50\%) mentioned Syria.

As Table 1 shows, users in Europe and North America constituted the source of most of the tweets for which we were able to identify a location, unsurprising given the Western-centric 
geographical spread of Twitter users in general. ${ }^{5}$ European and North American users referenced the Paris attacks more frequently while users in Middle East and North Africa, South Asia and elsewhere in Asia, while less numerous in our dataset, tweeted more frequently about Syria. About two-thirds of tweets were posted by members of the public, and those users were more likely to mention the Paris than the Syria attacks. However, we found - albeit in small numbers - that mainstream media, NGOs, religious authorities, celebrity and commercial accounts were more likely to tweet about Paris and activistbloggers about Syria.

Table 1 - Location and Types of Actors Posting Tweets about Paris and Syria

\begin{tabular}{lccc}
\hline Location of source & Paris & Syria & $\begin{array}{c}\text { \% of } \\
\text { sample }\end{array}$ \\
\hline Europe & & & sacr \\
North America & $70 \%$ & $27 \%$ & $17 \%$ \\
Other Asia & $48 \%$ & $60 \%$ & $12 \%$ \\
South Asia (India, Pakistan, Sri Lanka, Nepal) & $34 \%$ & $79 \%$ & $5 \%$ \\
Middle East and North Africa & $49 \%$ & $64 \%$ & $2 \%$ \\
Other Africa & $63 \%$ & $48 \%$ & $2 \%$ \\
South America & $70 \%$ & $44 \%$ & $1 \%$ \\
Australasia & $71 \%$ & $33 \%$ & $1 \%$ \\
Unclear & $57 \%$ & $55 \%$ & $52 \%$ \\
& & & \\
Type of actor & Paris & Syria & $\%$ of \\
\hline Member of the public & & & sample \\
Alternative media / blogger / citizen journalist / activist account & $51 \%$ & $63 \%$ & $5 \%$ \\
Commercial / business account & $89 \%$ & $16 \%$ & $4 \%$ \\
Celebrity & $69 \%$ & $37 \%$ & $1 \%$ \\
Mainstream media corporate account & $78 \%$ & $44 \%$ & $>1 \%$ \\
Mainstream media personal/journalist account & $78 \%$ & $22 \%$ & $>1 \%$ \\
\hline
\end{tabular}

\footnotetext{
${ }^{5}$ The large percentage of tweets whose location was coded as "Unclear" is because either those users either did not write any content in the "Location" field of their Twitter profiles or did not provide sufficient information to identify their location.
} 


\begin{tabular}{lccc}
\hline Pundit / opinion-writer & $60 \%$ & $40 \%$ & $>1 \%$ \\
National or international politician, policymaker or public official & $75 \%$ & $25 \%$ & $>1 \%$ \\
Local police / intelligence & $50 \%$ & $50 \%$ & $>1 \%$ \\
Expert (academic, think tank, retired police etc.) & $100 \%$ & $0 \%$ & $>1 \%$ \\
Non-Governmental Organisation or charity representative & $85 \%$ & $31 \%$ & $>1 \%$ \\
Religious authority & $92 \%$ & $15 \%$ & $>1 \%$ \\
Other & $67 \%$ & $33 \%$ & $>1 \%$ \\
Unclear & $55 \%$ & $56 \%$ & $22 \%$ \\
\hline
\end{tabular}

Table 2 shows some further descriptive statistics on the content and the diffusion networks of tweets mentioning Paris and Syria. In both cases tweets were most likely to be textual not visual, but about one-fifth of tweets contained images. Tweets about Paris were twice as likely (18\% versus 9\%) than tweets about Syria to include links to other social media content. Screengrabs of visual tweets combining the Eiffel Tour with a peace symbol were popular shared directly via Twitter or through a link to image-sharing social network Instagram.

Table 2 - Types of Content Shared, Topics Mentioned, Degrees of Antagonism and Agonism, and Network Size of Tweets about Paris and Syria

\begin{tabular}{lccc}
\hline Type of content shared & Paris & Syria & All tweets \\
\hline Only text & $53 \%$ & $67 \%$ & $59 \%$ \\
Images & $23 \%$ & $20 \%$ & $22 \%$ \\
Other social media & $18 \%$ & $9 \%$ & $14 \%$ \\
Other websites & $5 \%$ & $3 \%$ & $4 \%$ \\
Mainstream media & $1 \%$ & $1 \%$ & $1 \%$ \\
& & & \\
Topics mentioned & Paris & Syria & All tweets \\
Universalism & $19 \%$ & $18 \%$ & $17 \%$ \\
Justice and fairness & $8 \%$ & $27 \%$ & $15 \%$ \\
Foreign policy & $7 \%$ & $28 \%$ & $15 \%$ \\
Terrorists & $5 \%$ & $9 \%$ & $6 \%$ \\
Religion & $6 \%$ & $6 \%$ & $6 \%$ \\
Social media & $3 \%$ & $5 \%$ & $4 \%$ \\
\hline
\end{tabular}




\begin{tabular}{llll}
\hline Mainstream media & $2 \%$ & $4 \%$ & $2 \%$ \\
Migration & $1 \%$ & $3 \%$ & $2 \%$
\end{tabular}

$\begin{array}{lccc}\text { Agonism and antagonism } & \text { Paris } & \text { Syria } & \text { All tweets } \\ \text { Agonistic } & 14 \% & 41 \% & 24 \% \\ \text { Antagonistic } & 2 \% & 4 \% & 3 \%\end{array}$

\begin{tabular}{lccc} 
& Paris & Syria & All tweets \\
Mean retweets & 2.96 & 31.84 & 17.21 \\
Median retweets & 0 & 0 & 0 \\
Mean followers & 4751 & 3553 & 4373 \\
Median followers & 283 & 374 & 317 \\
Mean following & 1102 & 1317 & 1198 \\
Median following & 312 & 338 & 318 \\
\hline
\end{tabular}

Regarding topics mentioned, universalism was the most frequently mentioned topic across the whole dataset, equally present in both Paris and Syria tweets. However, there are striking differences in the extent to which those who posted about the two conflicts mentioned foreign policy and justice and fairness, of which there were three to four times more mentions in tweets about Syria than Paris. Tweets about Syria were also more likely, in smaller numbers, to mention mainstream media, migration and terrorism. The eight topics were entirely absent in $62 \%$ of tweets mentioning Paris, but only 33\% of tweets mentioning Syria. This indicates that the tweets about Syria were substantially more political than those about Paris, a majority of which was not relevant to any of the topics we are interested here. Most of the Paris tweets that we coded as 0 in all the topic categories feature expressions of solidarity and generic statements of feeling shocked or scared.

Our first hypothesis is therefore not supported here: The low occurrence of mentions of media, migration and terrorism suggests that the cycle of insecurity we expected to find was absent from discussions about Paris (only present in 7\% of Tweets) and was not particularly visible in those about Syria either (15\%). Users discussing Syria were concerned that France responded to the Paris attacks by intensifying its aerial military interventions in Syria. Users complained that the attacks on Paris received disproportionate attention from mainstream media, often expressed through sharing the image presented in Figure 2: 
Figure 2. World media framing of Paris (artist Lee Marej) ${ }^{6}$

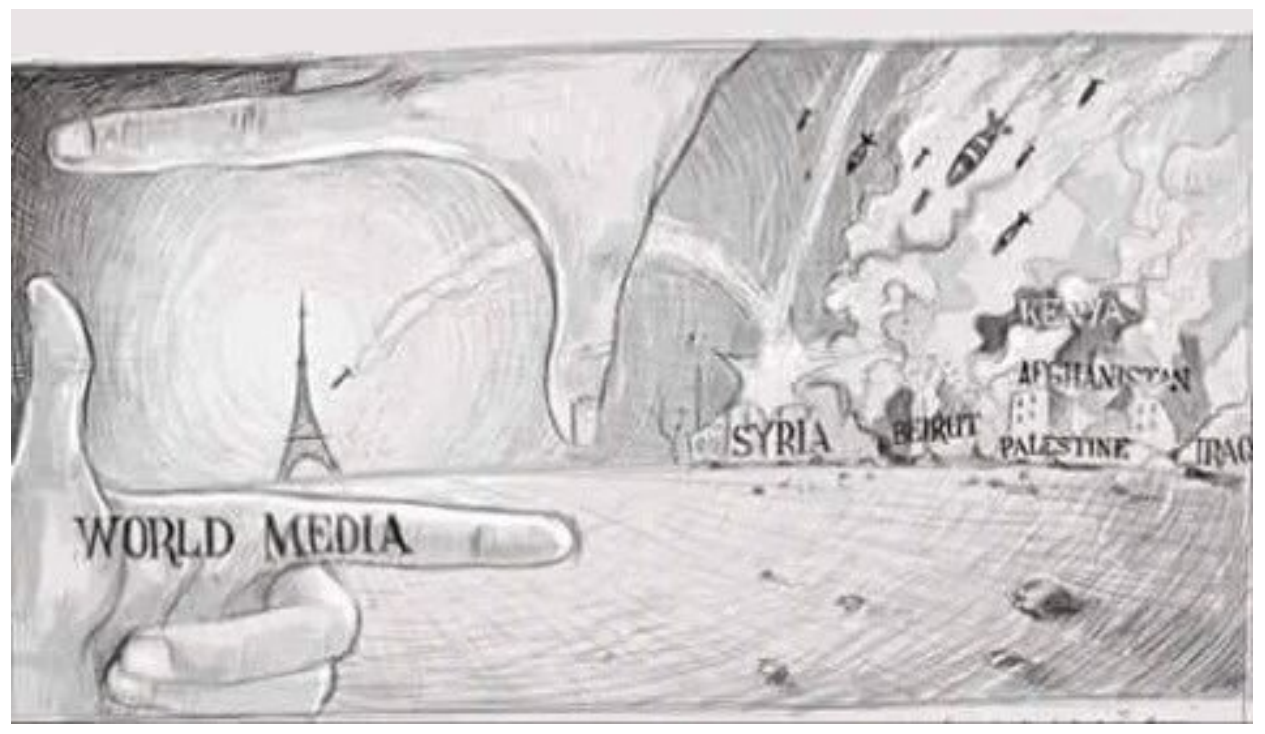

In Figure 2 we find a number of elements: attention to conflict in countries beyond Paris or Syria; foreign policy in the form of bombs falling from the sky; and world media directing attention exclusively on Paris, with the hands conveying a sense of human agency and culpability for this decision.

Tweets about Syria were more likely to be to either antagonistic or, by a substantial margin (41\% to 14\%), agonistic than those about Paris. With only 129 antagonistic tweets it was possible to read them as a sub-corpus after the coding. We found these 129 fell into groups: anti-Muslim tweets, most often from North America ('Lots of taqiyya [Sharia-approved lying] over on the \#PrayForSyria hashtag. Newsflash: The West doesn't fucking care about you primitive savages anymore.'); anti-racist tweets ('Racist pigs die \#PrayforSyria’); and users objecting to others posting \#PrayforParis on the grounds that France is a secular nation and it was religiosity that drove the terrorists ('I wish people would stop using \#prayforparis praying to some imaginary psychopath in the sky is the exact cause of this tragedy.'). While antagonistic tweets were the exception among all types of actors, tweets by activist-bloggers were most likely to be antagonistic (7.1 percent). The biographies of these antagonistic activist-bloggers contained hashtags such as \#ObamaLiedFrenchDied, \#MakeAmericaGreatAgain, \#ISaluteWhitePeople and \#ShiaGeonicide [sic]. However, given

\footnotetext{
${ }^{6}$ Few mainstream or social media users credited this artist and simply shared the image unattributed. The original is found at: http://leemarejlu.wix.com/leemarej
} 
the relatively small numbers of activist-bloggers, most antagonistic tweets came from members of the public. There were far more agonistic tweets and we explore this in the next section.

Tweets about Syria received on average substantially more retweets than those about Paris. However, as the medians in Table 2 show, more than half of those tweets received no retweet whatsoever-to be more precise, $77 \%$ of tweets about Paris and $68 \%$ of those mentioning Syria received no retweet. We also found different network characteristics in tweets about Paris and Syria. Users tweeting about Syria had a more even balance between the number of accounts following them and the number of accounts they followed (on average, 3,553 followers to 1,317 accounts followed) than users tweeting about Paris (mean of 4,751 to 1,101). However, these differences largely cancel out if we look at median values, where those who tweeted about Syria had actually more followers (374 versus 283) than those who tweeted about Paris. Given that we know Paris tweets received less retweets, we can characterise the conversation about Paris as a broad exchange, with wide reach and potential audience, but less retweeting intensity. The conversation about Syria was more close-knit, with a more even distribution of followers and followed, and more retweeting intensity.

\section{Analysis 2: Patterns of Agonism and Antagonism}

The conversation about Syria was more argumentative than that about Paris in terms of antagonism and, especially, agonism. Now we examine these patterns more systematically by looking at the correlations across all the content variables we measured, which we show in Table 3. The correlations range from -1 , which represents a perfect negative association, to +1 , which represents a perfect positive association. For practical purposes, we will focus only on coefficients that are higher than .1 or lower than -.1 and that are statistically significant. This provides a good indicator of the strength of the association between two variables, that is, how likely it was that tweets featuring topic A also featured topic B. 
Table 3 - Bivariate Correlations among Different Types of Contents of the Tweets

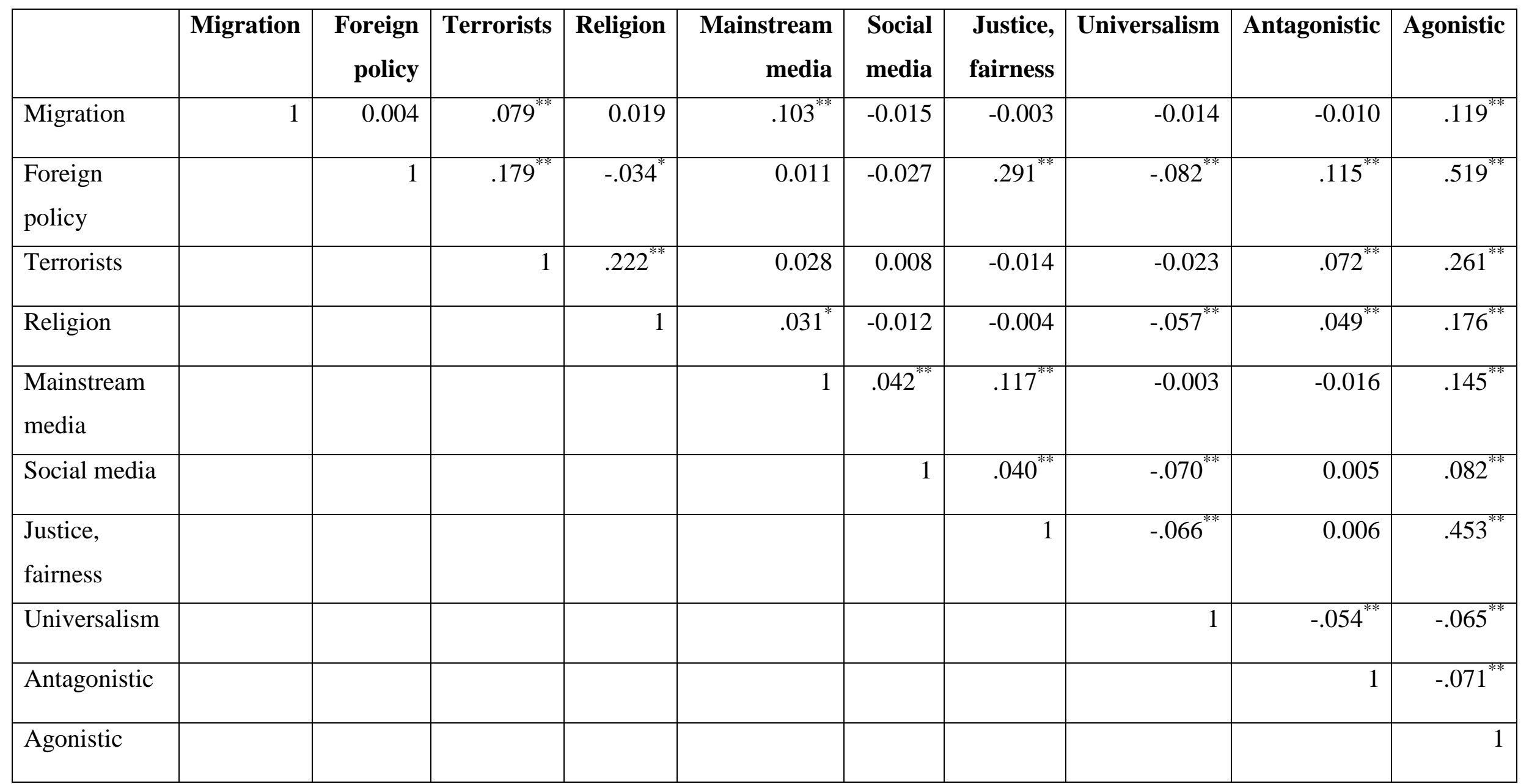

Note: cell entries are Pearson’s point-biserial correlations coefficients for dichotomous variables. 
** correlation is significant at $\mathrm{p} \leqslant .01$

* correlation is significant at $\mathrm{p} \leqslant .05$ 
The correlations in Table 3 point to a number of important patterns. First, tweets are far more likely to be agonistic on all topics except when mentioning universalism (the association with social media is also weak). In other words, to the extent that the tweets mentioned almost all of the political issues we coded, they were also likely to do so in argumentative fashion. Across the sample, users were more likely to be antagonistic only when tweeting about foreign policy, particularly users supporting French attacks on Syria, for example: 'DON'T \#PrayForSyria "If a spider bites you and runs away into a nest of spiders, how do you tell the spiders apart? DOESN'T MATTER!!”’ They were also more likely to be agonistic when discussing Syria, migration, foreign policy, religion, mainstream media, and justice and fairness.

This picture is reinforced by other clear associations. Discussion of mainstream media is strongly associated with discussion of migration and justice and fairness. This indicates criticism of journalists in their coverage of migrants and their focus on certain conflicts but not others. We also find a clear association between mentions of religion and terrorists, constituted by references to the religiosity of the Paris Attacks protagonists. Users either inferred a likely Islamic motivation or expressed a desire that not all Muslims be implicated in the actions of a few. However, regarding hypothesis one and the conflation of media, migration and terrorism, the numbers of these tweets are too small within the dataset to suggest that these narratives were as widespread as we expected. Far greater numbers of tweets linked foreign policy with terrorism and justice and fairness.

Universalism is far less likely to be expressed in discussions of foreign policy, when users discuss the French air strikes on Syria. Figure 3 below captures that sentiment:

Figure 3 - A bomb inscribed with 'From Paris with Love'

\footnotetext{
${ }^{7}$ This image is again without licence. News reports labelled it 'photoshopped' rather than assign credit to any photographer.
} 


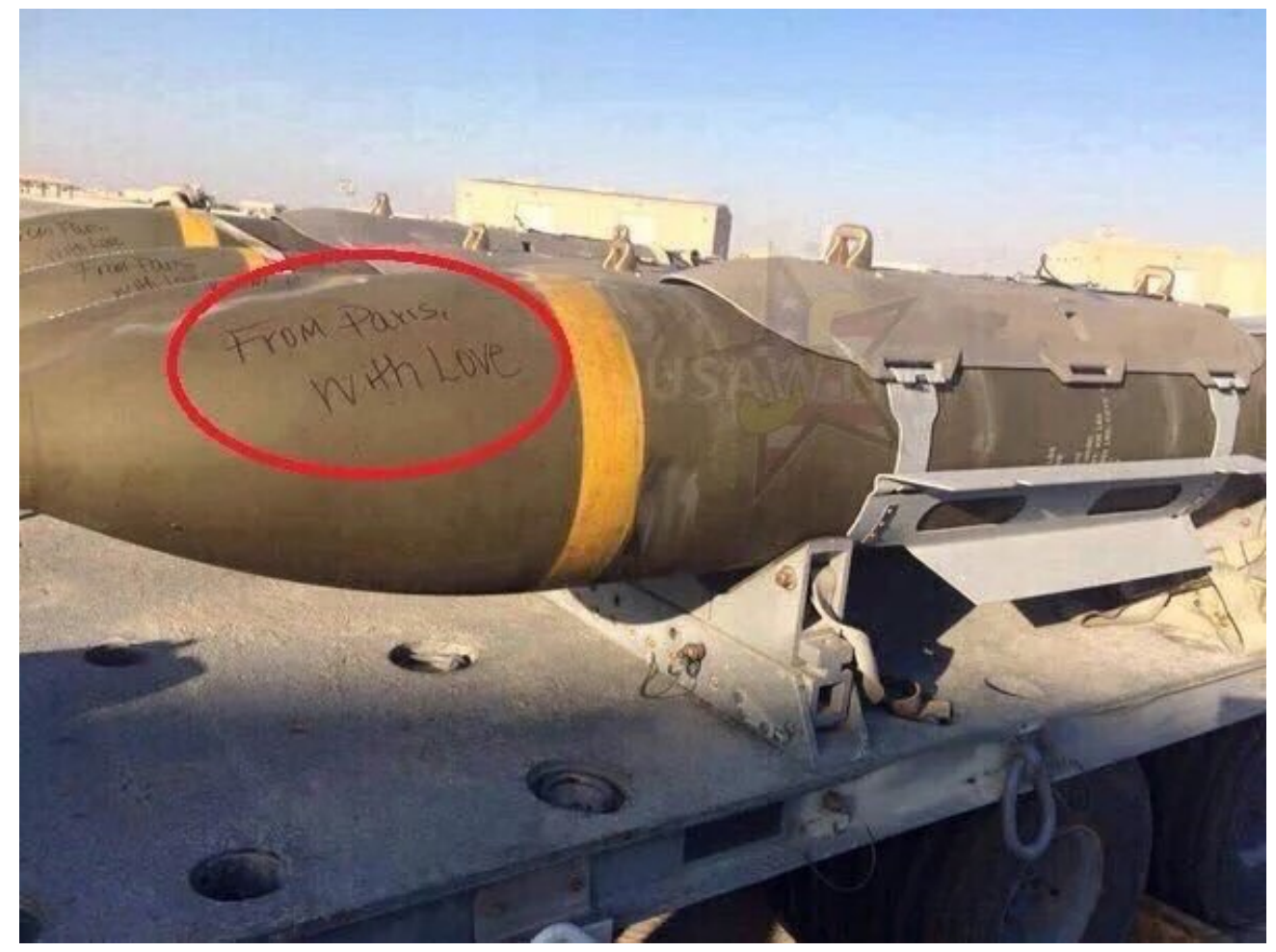

There was significant anger at the French government for launching airstrikes in response to the attacks. Many users posted statements along the lines of 'do not respond to violence with violence' or 'do not fight fire with fire'. Ultimately, the three strongest positive correlations in the entire dataset demonstrate that high agonism was constituted by statements about the French foreign policy (.519**) and justice and fairness (.453**), and those two topics, as we already saw, were also strongly correlated with one another (.291**). We have also already seen that tweets about Syria were substantially more likely to be antagonistic. Taken together, these findings suggest that the most compelling evidence we found for a cycle of insecurity has to do with insecurity generated by Western foreign policy rather than with insecurity deriving from Islamic terrorism.

Antagonistic tweets received a low mean of 3.22 retweets compared to a 17.3 retweet average for all tweets, but there was a noticeable difference between antagonistic tweets mentioning Paris, which had an average of 5.21 retweets, and antagonistic tweets mentioning Syria, which had an average of 3.74 retweets. In contrast, tweets that were agonistic received on average 13.1 retweets, but the difference between antagonistic tweets mentioning Paris and Syria was striking: while agonistic tweets about Paris were retweeted on average 4.45 times, agonistic tweets about Syria had on average 14.93 retweets. Agonistic tweets were often retweets and commented retweets of high profile critics of French foreign policy towards 
Syria such as Farhan Khan Kirk. Kirk describes himself in his Twitter biography as a blogger and social media activist. He has over 100,000 followers. He regularly posted graphics that articulated a policy critique, such as Figure 4. below. Kirk accompanied this graphic with the statement, '\#PrayForSyria Our enemy is not any religion, Our enemies are Corporates, The imperialists, The economic hitmen’. This was retweeted 400 times.

Figure 4 - Graphic in a Farhan Khan Kirk agonistic tweet of 16 November 2015

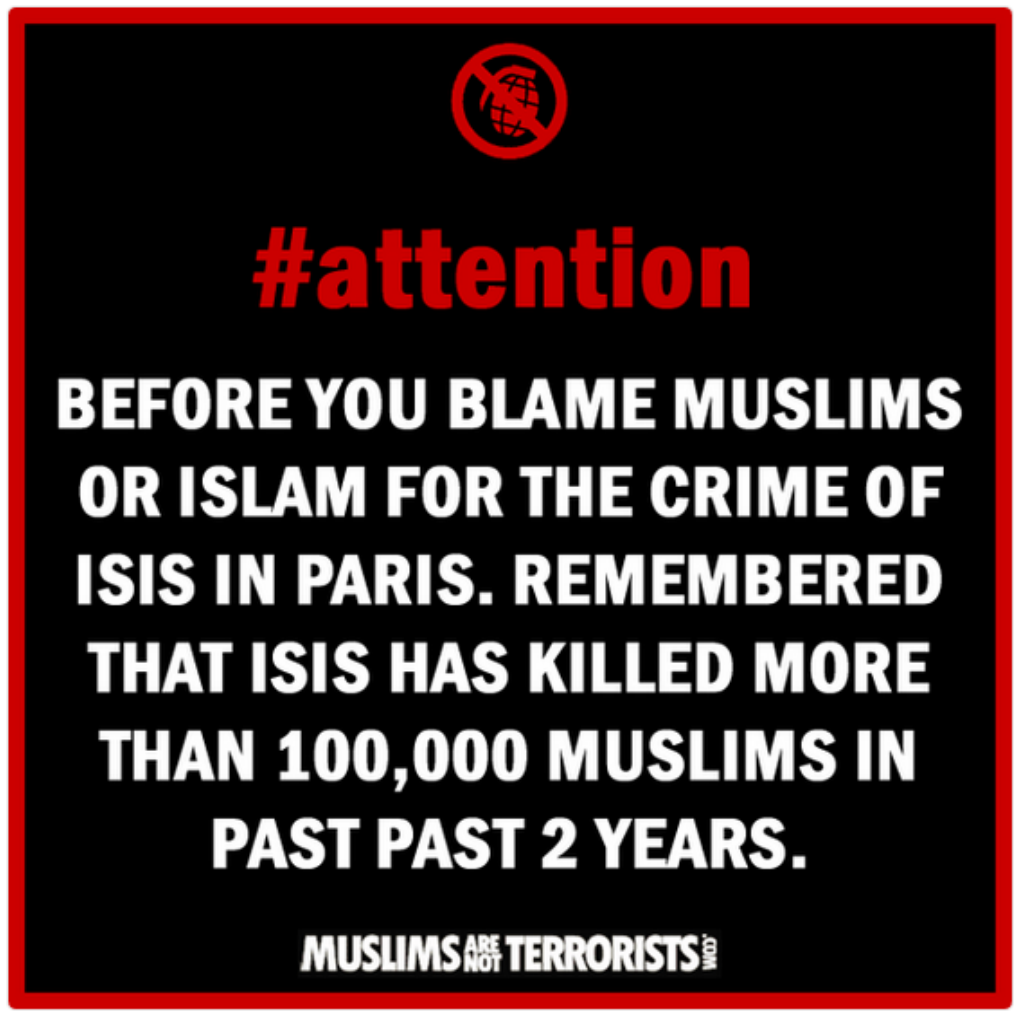

Responding to hypothesis two, this analysis suggests that antagonistic tweets do not attract retweets and drive conversation. However, whether a tweet is retweeted is more than simply a matter of whether it is agonistic or antagonistic. It also depends upon what topics it addresses, what content it conveys, how many followers its source has, and so forth. Only multivariate analysis can disentangle the role of these different factors, which we address in the next section.

Analysis 3: Do Agonism and Antagonism Drive Retweeting, and What Else Does? 
Table 4 shows the results of negative binomial regressions that predict the number of retweets obtained by each tweet in our dataset as a function of several independent variables. A negative binomial model is the most appropriate for the kind of data we are dealing with here, as the dependent variable is a count (the number of retweets) with very dispersed values and significant number of 0s, as highlighted earlier. We have built three different models: a first model with no interaction and two other models where we added interaction terms to capture different facets of the phenomena we are interested in and expand the analyses presented so far.

In Model 1, the dependent variable is estimated as a function of the type of tweet (differentiating between simple tweets, replies, and commented tweets, with all other tweets serving as reference category), the number of followers of the account at the time the tweet was posted (which we logged due to the high skewedness of its distribution), the type of content shared (with text-only tweets serving as reference category), what attacks were mentioned by the tweet, what topics it mentioned, and whether it was antagonistic or agonistic.

Results from Model 1 show that more retweets were likely from a straight tweet, by a user with more followers, containing images, and about Syria. Many of Farhan V. Kirk’s fitted this description. Second, more retweets were likely if a user mentioned religion and if the tweet was antagonistic. Such tweets contained statements along the lines of: Who are these monsters that did this? God will punish them. Or, why are you idiots praying, we are secular in France. Third, tweets were significantly less likely to be retweeted if they mentioned Paris, migration, the mainstream media, or social media. The latter tweets included those complaining about users only acting on social media or calling for prayers when "real" action was needed instead. Those kinds of criticism of other users were substantially unlikely to be shared by the users themselves.

Model 2 builds on Model 1 by adding interaction terms that differentiate between tweets mentioning Paris and Syria with respect to the content features we coded. Given that we found important differences in the topics and levels of agonism and antagonism of tweets pertaining to those two different conversations (see Table 2), did such differences in content also predict differences in the number of retweets a tweet received? The coefficients for the interaction terms show that tweets about migration, those discussing universalism, and those that mentioned the mainstream media were less likely to be retweeted when they were about 
Syria than when they were about Paris. By contrast, tweets about religion were significantly more likely to be retweeted when they mentioned Paris than when they mentioned Syria. This often involved users wishing to spread the idea that 'Muslims are not terrorists' after the Paris attacks. Tweets about social media show an interesting contrasting dynamic: if they mentioned Paris they were significantly more likely to be retweeted than if they mentioned Syria, but if they mentioned Syria they were significantly less likely to be retweeted than the average tweet. Users discussing Paris were more likely to share messages metacommunicating about the role and use of social media, for instance to promote symbolic action such as coordinated online silence or the propagation of images expressing solidarity, which elicited more retweets. The contrary was true for users discussing Syria, where discussions of social media were more likely to be centred on social media users' low interest for the plight of the Syrian people compared with the atrocities in Paris and elsewhere in the West, and these tweets were less likely to be retweeted. Interestingly, Model 2 also confirms that antagonistic tweets, albeit less numerous than agonistic ones in our sample, were more likely to be retweeted, but only if they were about Paris. Although tweets about Syria were generally more retweeted than those about Paris, all else being equal, antagonistic tweets about Paris received a greater marginal boost of retweets than antagonistic tweets mentioning Syria. This may be because of their rarity, or, on qualitative inspection, because they were retweeted frequently within tighter (North American) far right networks (see Analysis 1 above).

Finally, Model 3 also builds on Model 1 by adding four interaction terms that allow us to estimate whether tweets augmenting the moral panic-esque cycle of insecurity are more or less likely to be retweeted. With this set-up, the coefficient for "migration x terrorists”, for instance, allows estimation of how many retweets a tweet that features both migration and terrorists is predicted to achieve compared to a tweet that only features one of these two topics (while the coefficients for individual topics still capture the estimated number of retweets deriving from the presence of such topics). As can be seen from the last two columns of Table 4, tweets mentioning migration and terrorists, migration and mainstream media, and terrorists and mainstream media did not receive a significantly different number of retweets than tweets that only mentioned one of those topics. However, the fact that the coefficient for the three-way interaction between migration, terrorists, and mainstream media is positive and significant suggests that the conflation of those three topics, even if it only happened in a limited number of tweets, elicited a higher number of retweets than tweets 
mentioning both only one and two of the issues comprising the cycle of insecurity. Thus, although we found that discussions of those issues was too limited to claim that this cycle narrative emerged with the intensity we had expected, the evidence suggests that, even if the conflation of these topics did not happen frequently, when it did it was met by a noticeable user response in terms of the number of retweets those tweets received. 
Table 4 - Dependent Variable: Number of Retweets

\begin{tabular}{|c|c|c|c|c|c|c|}
\hline & \multicolumn{2}{|c|}{ Model 1} & \multicolumn{2}{|c|}{ Model 2} & \multicolumn{2}{|c|}{ Model 3} \\
\hline & $\mathbf{B}$ & Std. Error & $\mathbf{B}$ & Std. Error & $\mathbf{B}$ & Std. Error \\
\hline \multicolumn{7}{|l|}{ Type of tweet } \\
\hline Tweet & $1.703 *$ & .813 & $1.676^{*}$ & .802 & $1.683^{*}$ & .818 \\
\hline Commented tweet & .698 & .846 & .678 & .835 & .687 & .852 \\
\hline Reply & .460 & .958 & .500 & .945 & .440 & .963 \\
\hline Account followers (logged) & $.717^{* * *}$ & .054 & $.711^{* * *}$ & .048 & $.717^{* * *}$ & .054 \\
\hline \multicolumn{7}{|l|}{ Type of content shared } \\
\hline Mainstream media & -.702 & .492 & -.604 & .473 & -.753 & .497 \\
\hline Other websites & -.172 & .646 & -.160 & .641 & -.166 & .648 \\
\hline Social media & -.404 & .212 & -.354 & .219 & $-.421 *$ & .213 \\
\hline Images & $2.447 * * *$ & .318 & $2.365 * * *$ & .268 & $2.452 * * *$ & .317 \\
\hline \multicolumn{7}{|l|}{ Attacks mentioned } \\
\hline Paris & $-.611 * *$ & .222 & $-1.114 *$ & .447 & $-.617^{* *}$ & .223 \\
\hline Syria & $.749 * * *$ & .228 & $.600 * *$ & .203 & $.745^{* * *}$ & .227 \\
\hline Elsewhere & .442 & .229 & .316 & .226 & .440 & .229 \\
\hline
\end{tabular}


Issues mentioned in the tweet

Migration

Migration (Paris)

Foreign policy

Foreign policy (Paris)

Terrorists

Terrorists (Paris)

Religion

Religion (Paris)

Mainstream media

Mainstream media (Paris)

Social media

Social media (Paris)

Justice and fairness

Justice and fairness (Paris)

Universalism

Universalism (Paris)

$\begin{array}{cccccc}-1.376^{*} & .543 & -1.519 * * & .538 & -1.501^{*} & .606 \\ - & - & .566 & 1.277 & - & - \\ -.209 & .237 & -.044 & .260 & -.236 & .239 \\ - & - & -.421 & .399 & - & - \\ -.220 & .248 & -.328 & .261 & -.220 & .253 \\ - & - & .152 & .515 & - & - \\ .772 * * & .295 & -.606 & .323 & .774 * * & .295 \\ - & - & 1.824^{* * *} & .512 & - & - \\ -.951^{* *} & .315 & -.921 * & .376 & -1.021^{* *} & .330 \\ - & - & .146 & .568 & - & - \\ -1.210^{* * *} & .264 & -1.873^{* * *} & .391 & -1.189 * * * & .265 \\ - & - & 1.241^{*} & .490 & - & - \\ .148 & .222 & .049 & .260 & .145 & .223 \\ - & - & .251 & .375 & - & - \\ -.319 & .194 & -.663 * & .337 & -.329 & .195 \\ - & - & .649 & .375 & - & -\end{array}$

$\underline{\text { Cycle of fear associations }}$

Migration x Terrorists

$-$

- 2 -

$-1.342$

.818 


\begin{tabular}{|c|c|c|c|c|c|c|}
\hline Migration x Mainstream media & - & - & - & - & .664 & 1.420 \\
\hline Mainstream media x Terrorists & - & - & - & - & -1.562 & .898 \\
\hline Migration x Mainstream media x Terrorists & - & - & - & - & $4.547^{*}$ & 2.185 \\
\hline \multicolumn{7}{|l|}{ Rhetorical scope (?) of the tweet } \\
\hline Agonistic & .265 & .163 & .103 & .237 & .283 & .161 \\
\hline Agonistic (Paris) & - & - & .277 & .345 & - & - \\
\hline Antagonistic & $1.142 * *$ & .443 & .136 & .476 & $1.154^{* *}$ & .444 \\
\hline Antagonistic (Paris) & - & - & $1.659 *$ & .782 & - & - \\
\hline Intercept & $-6.486 * * *$ & .858 & $-6.039 * * *$ & .837 & $-6.458 * * *$ & .861 \\
\hline Chi square & 30842.43 & & 25694.95 & & 3043.57 & \\
\hline Log likelihood & -5131.81 & & -5108.02 & & -513.07 & \\
\hline
\end{tabular}




\section{Conclusions}

An examination of tweets containing \#PrayforParis and \#PrayforSyria in the aftermath of the November 2015 Paris Attacks, operationalising complex and fuzzy concepts from political and social theory like justice, universalism, agonism and antagonism, has allowed us to explain the dynamics of these Twitter conversations. We aimed to identify and explain the main differences in the contents, sources, and diffusion networks of the tweets about the Paris and Syria attacks. We hypothesized first that agonism and antagonism would be driven by a conflation of the topics media, migration and terrorism and, second, that antagonism is associated with retweet volumes. In Analysis 1 and 2 we found little conflation of media, migration and terrorism; instead, users discussed Syria, foreign policy, and justice and fairness. We found antagonism in relatively few tweets but agonism and antagonism occurred more frequently in discussion of Syria. Analysis 3 explained the likely retweet rate of tweets not just as a matter of whether they were agonistic or antagonistic but accounting for other aspects of the tweet, user and network. Again we find small but interesting findings. While there are more retweets about Syria, an antagonistic tweet about Paris is relatively likely to achieve higher retweets. This points to important network characteristics among those tweeting aggressively about Paris and the need for revenge, or those furious about French air strikes on Syria. Similarly, the conflation of media, migration and terrorism was infrequent but when it did occur, retweets were high.

What story does this leave us with? The Twitter conversations around \#PrayforParis and \#PrayforSyria cannot be reduced either to the contestation of global attention or a mediadriven cycle of insecurity. Tweets about social or mainstream media were not widely shared and links to news rare. Universalism was the most frequently mentioned theme. Users in all parts of the world engaged on all topics to some degree. Only in small networks within and across the two hashtag conversations did antagonism occur. In fact, the high degree of agonistic and non-visual tweets, particularly around Syria, suggests a robust exchange of claims and counter-claims. This is hardly the dystopian narrative of Twitter as a space of superficial memes and anonymous hate.

This study opens up our horizons for considering political communication not only concerning Paris and Syria, Europe and the Middle East, but about how transnational connectivity functions around major events featuring conflict and suffering. First, research 
must explain how visual communication has strategic effects. In Analysis 3 we found that, all else being equal, visual tweets receive more retweets, in particular if about the injustice or malign consequences of foreign policy. Users present and share photographs of victims: dead children or children crying over their dead parents, crying but mute to us, figures of absolute vulnerability because they lack all agency. Users describe 'looking at those children ... made me cry'. We experience not terror - an urge to flee - but horror: paralysis, repugnance, and isolation before the text (Cavarero, 2011). Bodies dismembered by bombs: the destruction of a person's figural unity. What is the political strategy in sharing these photographs and eliciting horror? Is it to remind us that every day people do horrible things (Sontag, 2003); that our government's bombing has horrible effects? Is the strategy to provoke questions rather than close reflection down? If so, the high degree of agonism present in these tweets suggests the strategy worked.

Second, we must think through ethical questions about what constitutes adequate or proper understanding of distant others' lives for us to respond as citizens. Problems loom concerning how ethics work in a global digital ecology and what Floridi labels the declining 'right to ignore'. In some cases, thanks to smartphone video, tweets allowed us to hear survivors tell their stories and perhaps sense their full personhood. Often, subtitles were added. In the telling of their story, the survivors move from being a generic category, a what - potential migrants or terrorists to some, as we saw in antagonistic tweets - to a specific person, a who; their lives are narratable on their own terms (Cavarero, 2000 [1997]). However, does this create a different relation to the event for global audiences? Given these children are never named, will audiences ever hear from that specific child again and hear the rest of their story? Does a "whole” story require a mix of biography and autobiography, and thus a traditional journalist to narrate the name and context, and conduct follow-up interviews months and years later? If so, then the absence of links to mainstream media in our sample suggests this does not occur on Twitter. Furthermore, do the children themselves have control over their own representation? Do they even know their testimony is being shared on Twitter? Who checks whether the subtitles are accurate? Does their individuality matter at all, or are their videos only shared by Twitter users because their situation is taken to be typical, representing a collective suffering, metonymic both for individuals not in the frame and for the entire social body, tied to the label "Syrians" (cf. Gürsel, 2016)? 
Building on this, third, we must confront the ethical dimensions of our methodological choices. There may have been fewer \#PrayforSyria tweets due to lower internet penetration in North Africa and Middle Eastern countries yet the sentiments expressed therein may have been present via other mediums. A single-medium study necessarily omits the wider media ecology within which political claims are expressed and therefore omits those claims from public scholarship. It also omits how dynamics on that medium interact with dynamics on others, for instance how \#PrayforSyria may have moved through broadsheet newspapers, Facebook and face-to-face conversations before reaching Twitter. Researchers must devise multi-method designs to capture these dynamics if the full richness of agonism, antagonism and the political are to be captured and explained.

\section{References}

Ampofo, L., Collister, S., O’Loughlin, B., \& Chadwick, A. (2015). Text Mining and Social Media: When Quantitative Meets Qualitative and Software Meets People. Peter Halfpenny and Rob Proctor (eds.) Innovations in Digital Research Methods, London: Sage, 161-91. Barnett, C. (2003). Culture and Democracy: Media, Space and Representation. Edinburgh: Edinburgh University Press.

Bennett, W. L., Foot, K., \& Xenos, M. (2011). Narratives and network organization: A comparison of fair trade systems in two nations. Journal of Communication, 61(2), 219-245. Berry, M., Garcia-Blanco, I., \& Moore, K. (2015) Press Coverage of the Refugee and Migrant Crisis in the EU: A Content Analysis of Five European Countries.

Report prepared for the United Nations High Commission for Refugees, December. Available at: www.unhcr.org/56bb369c9.pdf [Accessed 25 July 2016]

Boltanski, L. (1999). Distant suffering: Morality, media and politics. Cambridge: Cambridge University Press.

Burnap, P., \& Williams, M. L. (2016). Us and them: identifying cyber hate on Twitter across multiple protected characteristics. EPJ Data Science, 5(1), 1-15.

Cavarero, A. (2000) [1997] Relating Narratives: Storytelling and selfhood, London and New York: Routledge.

Cavarero, A. (2011) [2007] Horrorism: Naming Contemporary Violence, New York: Columbia University Press.

Cohen S. (1972). Folk Devils and Moral Panics. Oxford: Blackwell. 
Crilley, R. (2006) The Visual Politics of Legitimation in the Digital Age: The Cases of the British Army and the Syrian Opposition. Unpublished PhD thesis. University of Birmingham. Floridi, Luciano. (2014). The Fourth Revolution: How the Infosphere is Reshaping Human Reality. Oxford: Oxford University Press.

Freelon, Deen, McIlwain, Charlton D. and Clark, Meredith D. (2016) Beyond the Hashtags: \#Ferguson, \#Blacklivesmatter, and the online struggle for offline justice. Centre for Media \& Social Impact, American University, Washington, DC. Available at: http://www.cmsimpact.org/blmreport

Gerbaudo, P. (2016). Constructing Public Space| Rousing the Facebook Crowd: Digital Enthusiasm and Emotional Contagion in the 2011 Protests in Egypt and Spain. International Journal of Communication, 10, 254-273.

Gürsel, Z.D. (2016) Image Brokers: Visualizing World News in the Age of Digital Circulation, Oakland, CA: University of California Press.

Hall S., Critcher C., Jefferson T., Clarke C., and Roberts B. (1978). Policing the Crisis: Mugging, the State and Law and Order. London: Macmillan.

Hansen, L. K., Arvidsson, A., Nielsen, F. Å., Colleoni, E., \& Etter, M. (2011). Good friends, bad news-affect and virality in twitter. In Future information technology (pp. 34-43). Springer Berlin Heidelberg.

Laclau, E. and Mouffe, C. (1985). Hegemony and socialist strategy: towards a radical democratic politics. London: Verso.

Krippendorff, K. (2004). Content Analysis: An Introduction to Its Methodology. Second Edition. Thousand Oaks, CA: Sage.

Meraz, S., \& Papacharissi, Z. (2013). Networked Gatekeeping and Networked Framing on\# Egypt. The International Journal of Press/Politics, 18(2), 138-166.

Miller, Vincent. (2012). A Crisis of Presence: On-line culture and Being in the World. Space and Polity, 16(3), 265-285.

Mouffe, C. (2000). The Democratic Paradox. London: Verso.

Mouffe, C. (1992a). Preface: Democratic Politics Today. In Chantal Mouffe (ed.) Dimensions of radical democracy: pluralism, citizenship, community, London: Verso, 225-239.

Mouffe, C. (1992b). Democratic citizenship and the political community. In Chantal Mouffe (ed.) Dimensions of radical democracy: pluralism, citizenship, community, London: Verso, 225-239.

Neuendorf, K. A. (2002). The content analysis guidebook. Thousand Oaks, California: Sage Publications. 
Poynting, S., Noble, G., Tabar, P. and Collins, J. (2004). Bin Laden in the Suburbs. Sydney: Sydney Institute of Criminology.

Schmitt, C. (1976). The Concept of the Political. Trans. George Schwab. New Brunswick, NJ: Rutgers University Press.

Sontag, S. (2003) Regarding the Pain of Others, New York: Farrar, Straus, and Giroux. Warner, M. (2002). Publics and Counterpublics. Public Culture, 14(1), 49-90.

Williams, M. L., \& Burnap, P. (2015). Cyberhate on social media in the aftermath of Woolwich: A case study in computational criminology and big data. British Journal of Criminology, 56(2), 211-238. 


\section{Appendix: Code frame and intercoder reliability scores}

(A) TYPE OF ACTOR (Krippendorff's alpha $=.692$, achieved at $3^{\text {rd }}$ iteration)

1. Mainstream media corporate account

2. Mainstream media personal/journalist account

3. Alternative media / blogger / citizen journalist account

4. Pundit / opinion-writer

5. National or international politician, policymaker or public official

6. Local police / intelligence

7. Expert (academic, think tank, retired police etc)

8. Non-Governmental Organisation (NGO) or charity representative

9. Religious authority

10. Member of the public

11. Bot

12. Unclear

13. Account no longer available

14. Other

(B) LOCATION OF ACTOR (identified solely on the basis of the "Location" information provided by users in their Twitter profile; no reliability tests were conducted for this variable as it simply involves converting geographical information)

1. Europe

2. Middle East

3. Africa

4. North America

5. South America

6. Asia

7. Australasia

8. Unclear

(C) TYPES OF TWEET (Krippendorff's alpha $=.721$, achieved at $2^{\text {nd }}$ iteration)

1. Tweet - no user mentions and not a RT

2. Retweet (RT)

3. Modified tweet (MT) 
4. Reply (@ mention)

5. Other / unclear

(D) TYPE OF CONTENT SHARED (Krippendorff's alpha $=.810$, achieved at $2^{\text {nd }}$ iteration)

1. Mainstream media

2. Other websites

3. Other social media

4. Images

5. Only text

(E) ATTACKS MENTIONED (all coded as Yes/No)

1. Paris (Krippendorff's alpha $=.869$, achieved at $1^{\text {st }}$ iteration)

2. Syria (Krippendorff's alpha $=.961$, achieved at $1^{\text {st }}$ iteration)

3. Elsewhere (Krippendorff's alpha $=.942$, achieved at $3^{\text {rd }}$ iteration)

(F) TOPICS MENTIONED (all coded as Yes/No)

1. Migration (percent agreement $=99 \%$, achieved at $1^{\text {st }}$ iteration)

2. Foreign policy (Krippendorff's alpha $=.671$; percent agreement $=94 \%$, achieved at $1^{\text {st }}$ iteration)

3. Terrorists (percent agreement $=94 \%$, achieved at $2^{\text {nd }}$ iteration)

4. Religion (Krippendorff's alpha $=.692$; percent agreement $=96 \%$, achieved at $2^{\text {nd }}$ iteration)

5. Mainstream media (Krippendorff's alpha $=.754$; percent agreement $=98 \%$, achieved at $1^{\text {st }}$ iteration)

6. Social media (percent agreement $=96 \%$, achieved at $2^{\text {nd }}$ iteration)

7. Justice and fairness (percent agreement $=92 \%$, achieved at $1^{\text {st }}$ iteration)

8. Universalism (Krippendorff's alpha $=.774$; percent agreement $=91 \%$, achieved at $3^{\text {rd }}$ iteration)

(G) AGONISM AND ANTAGONISM (all coded as Yes/No)

1. Is this tweet antagonistic? (percent agreement $=93 \%$, achieved at $2^{\text {nd }}$ iteration)

2. Is this tweet agonistic? (Krippendorff's alpha $=.751$; percent agreement $=91 \%$, achieved at $3^{\text {rd }}$ iteration) 